\title{
Thanks to our reviewers in 2016
}

(c) Springer-Verlag Wien 2017

We would like to thank the following reviewers for their support by taking part in the peer-review process of the journal Amino Acids, The Forum for Amino Acid,
Peptide and Protein Research in 2016. Their contribution and advice are essential to maintain the high quality of the journal.
G. P. Ables
R. Ben
I. Abreu
H. Ben-Mabrouk
A. Agoulnik
L. Benov
G. Agüero-Chapin
C. Bergamini
K. Akerman
H.-G. Bernstein
W. Akers
M. Berry
S. Aksu
M. Bešter-Rogač
R. Alcazar
A. Bhunia
P. Bienert
R. T. Alexander
M. Binelli
A. Alpar
C. G. Bochet
A. Amaral
J. Bode
A. Anadón
C. Boesch
S. Annalisa
E. Bossi
T. Anthony
D. Bowles
J. Antonio
O. Braissant
C. Aragao
M. Brandsch
E. Aranda
M. Aronoff
T. Brigaud
S. Broeer
M. Aschi
M. Brosnan
M. Ashiuchi
E. Budzisz
C. Ashworth
O. Bussolati
K. Babij
P. Campos-Ferraz
T. Baczek
E. Candi
H. Cao
M. Bai
P. Caravan
G. Baldassarre
E. Carneiro
M. Bartoli
O. Carugo
J. Beltowski
R. Casadio
R. A. Casero Jr.
G. Cebrian-Torrejon
M. Cervelli
K. Chandra
D. Channe Gowda
M. Chattopadhyay
Z. Chen
K. Chen
S. Chevalier
M. Chinappi
V.-A. Chouinard
T. Churchward-Venne
E. Cilli
J. Ciriello
G. Clayton
N. Clebis
D. Columbus
J. Coronas
M. Courtney
P. Czaplewska
R. Danesi
G. Das
G. Dawe
S. Dayal
J.-P. De Bandt
B. G. de la Torre
A. De Leon Rodriguez
S. De Luca
W. De Malsche 
B. De Souza

P. Desogere

R. Dhaher

E. Dillon

R. Dobrowolski

T. Donovan

K. Drescher

J. Eckerson

A. Elshorbagy

C. Esposito

M. Estevez

D. Feith

L. Fesus

F. Flamigni

T. Fleming

A. Francesco

R. A. Fricker

N. Fujii

S. Fujiwara

M. Fändrich

V. Ganapathy

X.-H. Gao

Y. Ge

E. Gerner

J. Ghosh

S. Gilmour

J. Glazier

D. Goberdhan

S. Goel

E. Gonzales

E. Gonzalez

Y. Goto

S. Greenwood

A. Grubb

B. Gualano

R. Guzun

R. Głowacki

K. Hamase

E. Hanff

T. Hano

H. Hara

T. Harmand

J. Hartgerink

G. Hasko

S. Hinderlich

W. Hoehenwarter

J. Hoffman

H. Homma

S. Hu

Z. $\mathrm{Hu}$

M. Hubal
J. Hubert

C. Huck

W. Hunter

C. Hutton

F. Iannotti

R. Ientile

D. Ingrosso

D. Iosifescu

T. Ito

D. Jacobsen

K. Jain

C. Janke

E. Jankowska

E. Jimenez-Mareos

J. Jorrin-Novo

M. Kaartinen

A. Kaiser

A. Kajava

M. Kamihira

H. Kan

C. Kaufmann

E. Kawahara

J. Keillor

E. Keimpema

T. Keinänen

G. Kemp

S.-Y. Kim

C. Kim

T. Kimura

N. Kishore

M. Klapa

D. Knappe

T. Kobiela

D. Kodrík

J. Koehbach

L. Komsta

X. Kong

M. Kornacker

M. Kotter

W. Kraemer

R. Kreider

N. Krishnan

W. Kruger

A. Kuan

G. S. Kumar

I. Lambert

H.-S. Lee

E. Levy

F. Li

$\mathrm{K}$. Lindfors

H. Liu
S. Liu

Y. Liu

K. Lohner

A. Loizzo

G. Lombardi

C. $\mathrm{Lu}$

A. Lucia

W. L'Amoreaux

Q. Ma

N. Ma

R. Maas

M. T. Machini

P. J. Magistretti

G. Maier

K. Malenczyk

M. Manzanera

G. Mao

L. Mariniello

R. Marion-Letellier

P. L. Martelli

C. Masclaux-Daubresse

T. Matsui

H. Matsumoto

R. Maugham

J. Mayer

A. McAfee

G. Meca

N. Metanis

A. Meyer

Y. Miao

J. Miao

J. Miller

I. Miller

T. Mindt

C. Mischiati

T. Miyazaki

E. Moczydlowski

C. Moore

A. Mourier

A. Mozzarelli

H. Mukai

T. Nakanishi

B. Natalini

K. Niederreiter

G. Noctor

K. Nosaka

P. Nunn

N. Oshiro-Rapley

Y. Pan

E. Park

Y. M. Park 
H. Pasantes-Morales

S. Pasiakos

M. B. Passani

V. Pattabiraman

A. Pautz

G. Pejler

R. Penafiel

A. Peracchi

J. Perla-Kajan

D. Petras

J. Phang

G. Piccialli

W. Pichayakorn

T. Piera

M. Pietzsch

A. Pilc

D. Pinto

D. Piovesan

A. Pitarch

T. Pizzorusso

F. Polticelli

G. Porter

J. Prompers

M. Przybylski

U. Quitterer

S. Ramamoorthy

K. Rand

T. Rao

M. Ratcliffe

S. Rebuffat

J. P. Richie

S. Riechman

S. Rinaldi

S. Roberts

M. Robinson

C. Rockman-Greenberg

A. L. Rodrigues

B. Rogers

F. Rohrbacher

S. Rooney

D. Rosemberg

J. Rosene

K. J. Rosengren

J. S. Rudra

M. Ruiz

E. Sabido

K. Sahlin

F. Saito

Y. Sakaguchi

G. Salomons
M. Sani

A. Sasse

K. Scherf

H. Schlüter

F. Schmidt

B. Schmidt

M. Schouten

A. Schulze

K. Schwab

F. Seebeck

D. Sewell

F. Shabanpoor

Y. Shai

L. Shantz

A. Sharma

C. Shi

B. Shin

M. Sikora

T. Silva

P. Simek

M. Skelton

S. Smith

L. Sollid

M. Spetea

R. Srinivasagan

K. S. Srivenugopal

V. Stoven

R. Strongin

E. Szodorai

Z. Szondy

F. Sánchez-Jiménez

O. Tabbene

C. Tabolacci

H. Taegtmeyer

K. Takano

Y. Takemoto

B. Tan

S. Tanaka

P. Taylor

M. Teixido

D. Telci

J. Terrill

D. Thwaites

H. Tokumitsu

A. Toninello

F. Tsofliou

$\mathrm{Z}$. Tu

M. Turner

C. Turner

D. P. Turner

T. Uemura
N. Umezawa

A.-M. van Dam

F. J. van Spronsen à

E. Vandermarliere

R. Vaughan

M. Vendelin

E. Verderio

F. Verrey

F. Vianello

P. Vorkas

H. Wallace

H.-R. Wang

K. Wang

H. Wang

T. War

P. Watt

H. Wennemers

S. Wienkoop

B. Wieringa

B. Wieringa

H. Wolosker

Z. Wu

R. Wu

D. $\mathrm{Wu}$

J.-Y. Wu

M. Wyss

G. Węgrzyn

P. Xie

K. Yamada

Y.-B. Yan

L. Yang

Y. Yin

J. Zahiri

N. Zaki

A. Zakrzewicz

C. Zanelli

T. Zang

M. Zawada

W. Zeng

E. Zerovnik

S. Zervou

M. Zhang

Z. Zhang

S. Zhi

Z. Zhong

Z. Zhou

P. Zhou

R. Zhou 